\title{
A Multiple Attribute Decision Making Method Based on Uncertain Linguistic Heronian Mean
}

\author{
Xiaodi Liu, ${ }^{1,2}$ Jianjun Zhu, ${ }^{1}$ Guodong Liu, ${ }^{1,3}$ and Jingjing Hao' \\ ${ }^{1}$ College of Economics and Management, Nanjing University of Aeronautics and Astronautics, \\ Nanjing 211106, China \\ ${ }^{2}$ School of Mathematics and Physics, Anhui University of Technology, Ma'anshan 243002, China \\ ${ }^{3}$ Logistics School, Linyi University, Linyi 276000, China \\ Correspondence should be addressed to Xiaodi Liu; lxy1160@163.com
}

Received 2 August 2013; Accepted 24 August 2013

Academic Editor: Wei-Chiang Hong

Copyright (C) 2013 Xiaodi Liu et al. This is an open access article distributed under the Creative Commons Attribution License, which permits unrestricted use, distribution, and reproduction in any medium, provided the original work is properly cited.

\begin{abstract}
The Heronian mean is a useful aggregation operator which can capture the interrelationship of the input arguments. In this paper, we develop some Heronian means based on uncertain linguistic variables, such as the generalized uncertain linguistic Heronian mean (GULHM) and uncertain linguistic geometric Heronian mean (ULGHM), and some of their desirable properties are also investigated. Considering the different importance of the input arguments, we define the generalized uncertain linguistic weighted Heronian mean (GULWHM) and uncertain linguistic weighted geometric Heronian mean (ULWGHM). Then, a method of multiple attribute decision making under uncertain linguistic environment is presented based on the GULWHM or the ULWGHM. In the end, an example is given to demonstrate the effectiveness and feasibility of the proposed method.
\end{abstract}

\section{Introduction}

Multiple attribute decision making exists here and there, and a multiple attribute decision making problem is to find the most desirable candidate from some feasible alternatives. In real life, decision-makers often provide their preferences on alternatives using linguistic term sets instead of numerical values owing to the fuzziness of human thinking process, and multiple attribute decision making under linguistic environment is a focus in recent years [1-12]. In the process of decision making, the input arguments need to be aggregated by some proper approaches so that the decision makers can select the most desirable alternative. Among these approaches, the operators are widely used. Yager [13] introduced the ordered weighted averaging (OWA) operator, which has only been used in situations in which the input arguments are the exact numerical values. But now, it has been extended to accommodate linguistic environment $[2$, 14-17], uncertain linguistic environment [18-22], and some other preference representation structures [23, 24]. Uncertain linguistic variable, as a generalization form of linguistic variable, is more powerful in dealing with uncertainty than linguistic variable since it is characterized by a linguistic interval rather than a linguistic value. Since its appearance, the uncertain linguistic variable has received much attention from researchers. Based on the weighted arithmetic averaging (WAA) operator [25] and the ordered weighted averaging (OWA) operator [13], Xu [18] introduced some uncertain linguistic aggregation operators called uncertain linguistic weighted averaging (ULWA) operator, uncertain linguistic ordered weighted averaging (ULOWA) operator, and uncertain linguistic hybrid aggregation (ULHA) operator. The ULWA operator only weights the uncertain linguistic arguments while the ULOWA operator only weights the ordered positions of the uncertain linguistic arguments. The ULHA operator combines the advantages of the ULWA and the ULOWA operator and weights not only the given arguments but also their ordered positions. From a geometric point of view, $\mathrm{Xu}$ [20] proposed some uncertain linguistic aggregation operators, such as the uncertain linguistic geometric mean (ULGM), uncertain linguistic weighted geometric mean (ULWGM), and uncertain linguistic ordered weighted geometric (ULOWG) operator. In order to solve the drawbacks of the ULWGM and the ULOWG operator, Wei [21] developed 
the uncertain linguistic hybrid geometric mean (ULHGM) operator and proposed an approach to multiple attribute group decision making with uncertain linguistic information based on the ULWGM and ULHGM operators. In [22], Park et al. proposed the uncertain linguistic weighted harmonic mean (ULWHM) operator, uncertain linguistic ordered weighted harmonic mean (ULOWHM) operator, and uncertain linguistic hybrid harmonic mean (ULHHM) operator, and an illustrative example about determining the airconditioning system is also given to demonstrate the effectiveness and feasibility of the proposed method. Motivated by Yager and Filev [26], Xu [27] proposed some induced uncertain linguistic aggregation operators which can aggregate the decision making information in environments of mixing numeric and linguistic variables, such as the induced uncertain linguistic ordered weighted averaging (IULOWA) operator and the induced uncertain linguistic ordered weighted geometric (IULOWG) operator [20]. In [28], Xu generalized the IULOWA and the IULOWG operator and developed some generalized induced uncertain linguistic aggregation operators, including the generalized induced uncertain linguistic ordered weighted averaging (GIULOWA) operator and the generalized induced uncertain linguistic ordered weighted geometric (GIULOWG) operator.

However, the above uncertain linguistic aggregation approaches designed for solving multiple attribute decision making problems only consider the importance of the given arguments but ignore the correlation of them. Up to now, we are only aware of one paper on uncertain linguistic decision making that pays attention to the correlation of the input arguments [29]. In [29], Wei et al. utilized the uncertain linguistic Bonferroni mean (ULBM) operator and the uncertain linguistic geometric Bonferroni mean (ULGBM) operator which are an extension of the Bonferroni mean (BM) [30] to aggregate the uncertain linguistic arguments. The main advantage of the ULBM and ULGBM is that they can reflect the interrelationship of the input uncertain linguistic arguments. Nevertheless, these two means have their own disadvantages. For example, given a set of attributes $C_{i}(i=1,2, \ldots, n)$, the $\mathrm{BM}$ can reflect the correlation between any pair of attributes $C_{i}$ and $C_{j}(i \neq j)$ but neglect the relationship between the attribute $C_{i}$ and itself. Moreover, the BM considers the correlation between $C_{i}$ and $C_{j}(i \neq j)$ and the correlation between $C_{j}$ and $C_{i}(i \neq j)$ simultaneously, which results in potential redundancy. In order to solve these issues, we introduce the Heronian mean (HM) [31], the generalized Heronian mean $\left(\mathrm{GHM}_{1}\right)$ [32], and the geometric Heronian mean $\left(\mathrm{GHM}_{2}\right)[33]$ and extend them to accommodate uncertain linguistic environment.

To do so, the remainder of this paper is organized as follows. In Section 2, we briefly review some basic concepts, such as the uncertain linguistic variable, $\mathrm{HM}, \mathrm{GHM}_{1}$, and $\mathrm{GHM}_{2}$. In Section 3, we extend these means to accommodate the situation in which the input arguments are uncertain linguistic variables and develop some uncertain linguistic $\mathrm{He}-$ ronian means, such as generalized uncertain linguistic Heronian mean (GULHM), generalized uncertain linguistic weighted Heronian mean (GULWHM), uncertain linguistic geometric Heronian mean (ULGHM), and uncertain linguistic weighted geometric Heronian mean (ULWGHM). In Section 4, we propose a method for multiple attribute decision making with uncertain linguistic information based on GULWHM or ULWGHM. In Section 5, an example is given to verify the effectiveness and feasibility of the proposed method. Section 6 ends the paper with some concluding remarks.

\section{Uncertain Linguistic Variables and Heronian Mean}

2.1. Uncertain Linguistic Variables. Let $S=\left\{s_{i} \mid i=1,2, \ldots\right.$, $t\}$ be a linguistic term set with odd cardinality, where $s_{i}$ represents a possible value for a linguistic variable. For example, a set of seven terms, $S$, could be defined as follows:

$$
\begin{aligned}
S=\left\{s_{1}\right. & =\text { extremely poor, } s_{2}=\text { very poor, } s_{3}=\text { poor, } \\
s_{4} & =\text { medium, } s_{5}=\text { good, } s_{6}=\text { very good, } \\
s_{7} & =\text { extremely good }\} .
\end{aligned}
$$
21].

It is usually required that there exist the following $[7,17$,

(1) The set is ordered as $s_{i} \geq s_{j}$ if $i \geq j$.

(2) There is the negation operator $\operatorname{neg}\left(s_{i}\right)=s_{j}$ such that $i+j=t+1$.

(3) Max operator $\max \left(s_{i}, s_{j}\right)=s_{i}$, if $s_{i} \geq s_{j}$.

(4) Min operator $\min \left(s_{i}, s_{j}\right)=s_{i}$, if $s_{i} \leq s_{j}$.

To preserve all the given information, the discrete term set $S$ should be extended to a continuous term set $\bar{S}=$ $\left\{s_{\alpha} \mid s_{1} \leq s_{\alpha} \leq s_{q}, \alpha \in[1, q]\right\}$, where $q$ is a sufficiently large positive integer; if $s_{\alpha} \in S$, then we call $s_{\alpha}$ the original term; otherwise, we call $s_{\alpha}$ the virtual term $[17,21]$. The decision maker, in general, uses the original linguistic terms to evaluate alternatives, and the virtual linguistic terms can only appear in operations.

Definition 1 (see $[18-22,27,28]$ ). Let $\widetilde{s}=\left[s_{\alpha}, s_{\beta}\right]$, where $s_{\alpha}, s_{\beta} \in \bar{S}, s_{\alpha}$, and $s_{\beta}$ are the lower and the upper limits, respectively, and then we call $\widetilde{s}$ the uncertain linguistic variable. Suppose that $\bar{S}$ is the set of all uncertain linguistic variables.

If $s_{\alpha}=s_{\beta}$, then the uncertain linguistic variable $\widetilde{s}$ is reduced to a linguistic value. Consider any three uncertain linguistic variables $\widetilde{s}=\left[s_{\alpha}, s_{\beta}\right], \widetilde{s}_{1}=\left[s_{\alpha_{1}}, s_{\beta_{1}}\right], \widetilde{s}_{2}=\left[s_{\alpha_{2}}, s_{\beta_{2}}\right]$, and let $\lambda \in[0,1]$; then their operational laws are defined as follows $[18-21,27,28]$ :

(1) $\widetilde{s}_{1} \oplus \widetilde{s}_{2}=\left[s_{\alpha_{1}}, s_{\beta_{1}}\right] \oplus\left[s_{\alpha_{2}}, s_{\beta_{2}}\right]=\left[s_{\alpha_{1}} \oplus s_{\alpha_{2}}, s_{\beta_{1}} \oplus s_{\beta_{2}}\right]=$ $\left[s_{\alpha_{1}+\alpha_{2}}, s_{\beta_{1}+\beta_{2}}\right]$;

(2) $\lambda \widetilde{s}=\lambda\left[s_{\alpha}, s_{\beta}\right]=\left[\lambda s_{\alpha}, \lambda s_{\beta}\right]=\left[s_{\lambda \alpha}, s_{\lambda \beta}\right]$;

(3) $\widetilde{s}_{1} \otimes \widetilde{s}_{2}=\left[s_{\alpha_{1}}, s_{\beta_{1}}\right] \otimes\left[s_{\alpha_{2}}, s_{\beta_{2}}\right]=\left[s_{\alpha_{1}} \otimes s_{\alpha_{2}}, s_{\beta_{1}} \otimes s_{\beta_{2}}\right]=$ $\left[s_{\alpha_{1} \alpha_{2}}, s_{\beta_{1} \beta_{2}}\right]$;

(4) $\widetilde{s}^{\lambda}=\left(\left[s_{\alpha}, s_{\beta}\right]\right)^{\lambda}=\left[\left(s_{\alpha}\right)^{\lambda},\left(s_{\beta}\right)^{\lambda}\right]=\left[s_{\alpha^{\lambda}}, s_{\beta^{\lambda}}\right]$. 
Moreover, the following relationship can be easily proved:

(5) $\widetilde{s}_{1} \oplus \widetilde{s}_{2}=\widetilde{s}_{2} \oplus \widetilde{s}_{1}$;

(6) $\tilde{s}_{1} \otimes \widetilde{s}_{2}=\widetilde{s}_{2} \otimes \tilde{s}_{1}$;

(7) $\lambda\left(\widetilde{s}_{1} \oplus \widetilde{s}_{2}\right)=\lambda \widetilde{s}_{1} \oplus \lambda \widetilde{s}_{2}$;

(8) $\left(\widetilde{s}_{1} \otimes \widetilde{s}_{2}\right)^{\lambda}=\widetilde{s}_{1}^{\lambda} \otimes \widetilde{s}_{2}^{\lambda}$;

(9) $\lambda_{1} \widetilde{s} \oplus \lambda_{2} \widetilde{s}=\left(\lambda_{1}+\lambda_{2}\right) \widetilde{s}$;

(10) $\tilde{s}^{\lambda_{1}} \otimes \widetilde{s}^{\lambda_{2}}=\widetilde{s}^{\lambda_{1}+\lambda_{2}}$.

In order to compare the uncertain linguistic variables, we give the following definition.

Definition 2 (see [34]). Let $\widetilde{s}_{1}=\left[s_{\alpha_{1}}, s_{\beta_{1}}\right]$ and $\widetilde{s}_{2}=\left[s_{\alpha_{2}}, s_{\beta_{2}}\right]$ be two uncertain linguistic variables, and let len $\left(\widetilde{s}_{1}\right)=\beta_{1}-$ $\alpha_{1}$ and len $\left(\widetilde{s}_{2}\right)=\beta_{2}-\alpha_{2}$; then the degree of possibility of $\widetilde{s}_{1} \geq$ $\widetilde{s}_{2}$ is defined as

$$
p\left(\widetilde{s}_{1} \geq \widetilde{s}_{2}\right)=\frac{\max \left(0, \beta_{1}-\alpha_{2}\right)-\max \left(0, \alpha_{1}-\beta_{2}\right)}{\operatorname{len}\left(\widetilde{s}_{1}\right)+\operatorname{len}\left(\widetilde{s}_{2}\right)} .
$$

From Definition 2, we can easily get the following results:

(1) $0 \leq p\left(\widetilde{s}_{1} \geq \widetilde{s}_{2}\right) \leq 1,0 \leq p\left(\widetilde{s}_{2} \geq \widetilde{s}_{1}\right) \leq 1$;

(2) $p\left(\widetilde{s}_{1} \geq \widetilde{s}_{2}\right)+p\left(\widetilde{s}_{2} \geq \widetilde{s}_{1}\right)=1$. Especially, $p\left(\widetilde{s}_{1} \geq \widetilde{s}_{1}\right)=$ $p\left(\widetilde{s_{2}} \geq \widetilde{s_{2}}\right)=1 / 2$.

2.2. Heronian Mean. Heronian mean (HM), which is one of the aggregation methods, has the desirable characteristic that it can reflect the interrelationship of the input arguments. The definition of HM is as follows.

Definition 3 (see [31]). Let $a_{i}(i=1,2, \ldots, n)$ be a collection of nonnegative numbers. If

$$
\operatorname{HM}\left(a_{1}, a_{2}, \ldots, a_{n}\right)=\frac{2}{n(n+1)} \sum_{i=1}^{n} \sum_{j=i}^{n} \sqrt{a_{i} a_{j}},
$$

then HM is called the Heronian mean (HM).

Based on Definition 3, Yu and $\mathrm{Wu}[32,33]$ proposed the generalized Heronian mean $\left(\mathrm{GHM}_{1}\right)$ and the geometric Heronian mean $\left(\mathrm{GHM}_{2}\right)$.

Definition 4 (see [32]). Let $p, q \geq 0$ and $p, q$ do not take the value 0 simultaneously. Let $a_{i}(i=1,2, \ldots, n)$ be a collection of nonnegative numbers. If

$$
\operatorname{GHM}_{1}^{p, q}\left(a_{1}, a_{2}, \ldots, a_{n}\right)=\left(\frac{2}{n(n+1)} \sum_{i=1}^{n} \sum_{j=i}^{n} a_{i}^{p} a_{j}^{q}\right)^{1 /(p+q)},
$$

then $\mathrm{GHM}_{1}$ is called the generalized Heronian mean $\left(\mathrm{GHM}_{1}\right)$. If $p=q=1 / 2$ especially, then the $\mathrm{GHM}_{1}$ is reduced to HM.
It is noted that the $\mathrm{GHM}_{1}$ has the following properties:

(1) $\operatorname{GHM}_{1}^{p, q}(0,0, \ldots, 0)=0$;

(2) $\operatorname{GHM}_{1}^{p, q}(a, a, \ldots, a)=a$, if $a_{i}=a$, for all $i$;

(3) $\operatorname{GHM}_{1}^{p, q}\left(a_{1}, a_{2}, \ldots, a_{n}\right) \geq \operatorname{GHM}_{1}^{p, q}\left(b_{1}, b_{2}, \ldots, b_{n}\right)$, that is, $\mathrm{GHM}_{1}^{p, q}$ is monotonic, if $a_{i} \geq b_{i}$, for all $i$;

(4) $\min _{i}\left\{a_{i}\right\} \leq \operatorname{GHM}_{1}^{p, q}\left(a_{1}, a_{2}, \ldots, a_{n}\right) \leq \max _{i}\left\{a_{i}\right\}$.

Example 5. Let $a_{1}, a_{2}, a_{3}$ be three nonnegative numbers and $p=q=2$; then

$$
\begin{aligned}
\operatorname{GHM}_{1}^{p, q}\left(a_{1}, a_{2}, \ldots, a_{n}\right) \\
=\left(\frac{2}{n(n+1)} \sum_{i=1}^{n} \sum_{j=i}^{n} a_{i}^{p} a_{j}^{q}\right)^{1 /(p+q)} \\
=\left(\frac{1}{6}\left(a_{1}^{2} a_{1}^{2}+a_{1}^{2} a_{2}^{2}+a_{1}^{2} a_{3}^{2}+a_{2}^{2} a_{2}^{2}+a_{2}^{2} a_{3}^{2}+a_{3}^{2} a_{3}^{2}\right)\right)^{1 / 4} .
\end{aligned}
$$

If we use Bonferroni mean (BM) [30] to aggregate the above three nonnegative numbers, then

$$
\begin{aligned}
\operatorname{BM}^{p, q} & \left(a_{1}, a_{2}, \ldots, a_{n}\right) \\
= & \left(\frac{1}{n(n-1)} \sum_{\substack{i, j=1 \\
i \neq j}}^{n} a_{i}^{p} a_{j}^{q}\right)^{1 /(p+q)} \\
= & \left(\frac{1}{6}\left(a_{1}^{2} a_{2}^{2}+a_{1}^{2} a_{3}^{2}+a_{2}^{2} a_{1}^{2}+a_{2}^{2} a_{3}^{2}+a_{3}^{2} a_{1}^{2}+a_{3}^{2} a_{2}^{2}\right)\right)^{1 / 4} .
\end{aligned}
$$

From the above analysis, we can find that the BM computes $a_{1}^{2} a_{2}^{2}, a_{2}^{2} a_{1}^{2}, a_{1}^{2} a_{3}^{2}, a_{3}^{2} a_{1}^{2}, a_{2}^{2} a_{3}^{2}$, and $a_{3}^{2} a_{2}^{2}$ separately. However, $a_{1}^{2} a_{2}^{2}$ is equal to $a_{2}^{2} a_{1}^{2}, a_{1}^{2} a_{3}^{2}$ is equal to $a_{3}^{2} a_{1}^{2}$, and $a_{2}^{2} a_{3}^{2}$ is equal to $a_{3}^{2} a_{2}^{2}$. Hence, it results in potential redundancy. Moreover, the BM has not paid attention to $a_{1}^{2} a_{1}^{2}$, $a_{2}^{2} a_{2}^{2}$, and $a_{3}^{2} a_{3}^{2}$. Nevertheless, the $\mathrm{GHM}_{1}$ can solve the two problems effectively.

Definition 6 (see [33]). Let $p, q \geq 0$ and $p, q$ do not take the value 0 simultaneously. Let $a_{i}(i=1,2, \ldots, n)$ be a collection of nonnegative numbers. If

$$
\operatorname{GHM}_{2}^{p, q}\left(a_{1}, a_{2}, \ldots, a_{n}\right)=\frac{1}{p+q} \prod_{i=1, j=i}^{n}\left(p a_{i}+q a_{j}\right)^{2 / n(n+1)}
$$

then $\mathrm{GHM}_{2}$ is called the geometric Heronian mean $\left(\mathrm{GHM}_{2}\right)$.

It is noted that the $\mathrm{GHM}_{2}$ has the following properties:

(1) $\operatorname{GHM}_{2}^{p, q}(0,0, \ldots, 0)=0$;

(2) $\operatorname{GHM}_{2}^{p, q}(a, a, \ldots, a)=a$, if $a_{i}=a$, for all $i$; 
(3) $\operatorname{GHM}_{2}^{p, q}\left(a_{1}, a_{2}, \ldots, a_{n}\right) \geq \operatorname{GHM}_{2}^{p, q}\left(b_{1}, b_{2}, \ldots, b_{n}\right)$ that is, $\mathrm{GHM}_{2}^{p, q}$ is monotonic, if $a_{i} \geq b_{i}$, for all $i$;

(4) $\min _{i}\left\{a_{i}\right\} \leq \operatorname{GHM}_{2}^{p, q}\left(a_{1}, a_{2}, \ldots, a_{n}\right) \leq \max _{i}\left\{a_{i}\right\}$.

Example 7. Let $a_{1}, a_{2}, a_{3}$ be three nonnegative numbers and $p=q=1$; then

$$
\begin{aligned}
\operatorname{GHM}_{2}^{p, q} & \left(a_{1}, a_{2}, \ldots, a_{n}\right) \\
= & \frac{1}{p+q} \prod_{i=1, j=i}^{n}\left(p a_{i}+q a_{j}\right)^{2 / n(n+1)} \\
= & \frac{1}{2}\left(\left(a_{1}+a_{1}\right)\left(a_{1}+a_{2}\right)\left(a_{1}+a_{3}\right)\right. \\
& \left.\quad \times\left(a_{2}+a_{2}\right)\left(a_{2}+a_{3}\right)\left(a_{3}+a_{3}\right)\right)^{1 / 6} .
\end{aligned}
$$

If we use geometric Bonferroni mean (GBM) proposed by Xia et al. [35] to aggregate the above three nonnegative numbers, then

$$
\begin{aligned}
& \operatorname{GBM}^{p, q}\left(a_{1}, a_{2}, \ldots, a_{n}\right) \\
& =\frac{1}{p+q} \prod_{\substack{i, j=1 \\
i \neq j}}^{n}\left(p a_{i}+q a_{j}\right)^{1 / n(n-1)} \\
& =\frac{1}{2}\left(\left(a_{1}+a_{2}\right)\left(a_{1}+a_{3}\right)\left(a_{2}+a_{1}\right)\right. \\
& \left.\quad \times\left(a_{2}+a_{3}\right)\left(a_{3}+a_{1}\right)\left(a_{3}+a_{2}\right)\right)^{1 / 6} .
\end{aligned}
$$

Similar to BM, the GBM also results in potential redundancy. Furthermore, it has not paid attention to $\left(a_{1}+a_{1}\right),\left(a_{2}+\right.$ $\left.a_{2}\right)$, and $\left(a_{3}+a_{3}\right)$. However, the $\mathrm{GHM}_{2}$ can solve the two problems effectively.

\section{Uncertain Linguistic Heronian Means}

3.1. The GULHM and the GULWHM. The $\mathrm{GHM}_{1}$ has the desirable characteristic capturing the interrelationship of the input arguments. However, the arguments suitable to be aggregated by the $\mathrm{GHM}_{1}$ usually take the forms of nonnegative real numbers. In this section, we will extend the $\mathrm{GHM}_{1}$ to accommodate the situations in which the input arguments are uncertain linguistic variables. Based on the operational rules on uncertain linguistic variables and Definition 4, we give the generalized uncertain linguistic Heronian mean (GULWHM) in the following.

Definition 8. Let $p, q \geq 0$ and $p, q$ do not take the value 0 simultaneously. Let $\widetilde{s}_{i}=\left[s_{\alpha_{i}}, s_{\beta_{i}}\right](i=1,2, \ldots, n)$ be a collection of uncertain linguistic variables. If

$$
\begin{aligned}
& \operatorname{GULHM}^{p, q}\left(\widetilde{s}_{1}, \widetilde{s}_{2}, \ldots, \widetilde{s}_{n}\right) \\
& =\left(\frac{2}{n(n+1)} \bigoplus_{i=1, j=i}^{n}\left(\widetilde{s}_{i}^{p} \otimes \widetilde{s}_{j}^{q}\right)\right)^{1 /(p+q)}
\end{aligned}
$$

$$
\begin{aligned}
= & {\left[\left(\frac{2}{n(n+1)} \bigoplus_{i=1, j=i}^{n}\left(s_{\alpha_{i}}^{p} \otimes s_{\alpha_{j}}^{q}\right)\right)^{1 /(p+q)},\right.} \\
& \left.\left(\frac{2}{n(n+1)} \bigoplus_{i=1, j=i}^{n}\left(s_{\beta_{i}}^{p} \otimes s_{\beta_{j}}^{q}\right)\right)^{1 /(p+q)}\right],
\end{aligned}
$$

then the GULHM is called the generalized uncertain linguistic Heronian mean (GULHM). If $p=q=1 / 2$; then the GULHM reduces to

$$
\begin{aligned}
\operatorname{GULHM}^{1 / 2,1 / 2}\left(\widetilde{s}_{1}, \widetilde{s}_{2}, \ldots, \widetilde{s}_{n}\right) \\
=\frac{2}{n(n+1)} \bigoplus_{i=1, j=i}^{n}\left(\widetilde{s}_{i}^{1 / 2} \otimes \widetilde{s}_{j}^{1 / 2}\right) \\
=\left[\frac{2}{n(n+1)} \bigoplus_{i=1, j=i}^{n}\left(s_{\alpha_{i}}^{1 / 2} \otimes s_{\alpha_{j}}^{1 / 2}\right),\right. \\
\left.\frac{2}{n(n+1)} \bigoplus_{i=1, j=i}^{n}\left(s_{\beta_{i}}^{1 / 2} \otimes s_{\beta_{j}}^{1 / 2}\right)\right],
\end{aligned}
$$

which we call the uncertain linguistic Heronian mean (ULHM).

In the following, we investigate the desirable properties of the GULHM.

Theorem 9 (idempotency). Let $\widetilde{s}_{i}=\left[s_{\alpha_{i}}, s_{\beta_{i}}\right](i=1,2, \ldots, n)$ be a collection of uncertain linguistic variables. If all $\tilde{s}_{i}$ are equal, that is, $\widetilde{s}_{i}=\widetilde{s}=\left[s_{\alpha}, s_{\beta}\right]$ for all $i$, then

$$
\operatorname{GULHM}^{p, q}\left(\widetilde{s}_{1}, \widetilde{s}_{2}, \ldots, \widetilde{s}_{n}\right)=\widetilde{s} .
$$

Proof. Consider the following:

$$
\begin{aligned}
\operatorname{GULHM}^{p, q}\left(\widetilde{s}_{1}, \widetilde{s}_{2}, \ldots, \widetilde{s}_{n}\right) \\
=\operatorname{GULHM}^{p, q}(\widetilde{s}, \widetilde{s}, \ldots, \widetilde{s}) \\
=\left(\frac{2}{n(n+1)} \bigoplus_{i=1, j=i}^{n}\left(\widetilde{s}^{p} \otimes \widetilde{s}^{q}\right)\right)^{1 /(p+q)} \\
=\left[\frac{2}{n(n+1)} \bigoplus_{i=1, j=i}^{n}\left(s_{\alpha}^{p} \otimes s_{\alpha}^{q}\right),\right. \\
=\left[\frac{2}{n(n+1)} \bigoplus_{i=1, j=i}^{n}\left(s_{\beta}^{p} \otimes s_{\beta}^{q}\right)\right]^{1 /(p+q)} \\
\left.=s_{\beta}\right] .
\end{aligned}
$$


Theorem 10 (permutation). Let $\widetilde{s}_{i}=\left[s_{\alpha_{i}}, s_{\beta_{i}}\right]$ and $\vec{s}_{i}=$ $\left[s_{\alpha_{i}}^{\prime}, s_{\beta_{i}}^{\prime}\right](i=1,2, \ldots, n)$ be two collections of uncertain linguistic variables; then

$$
\operatorname{GULHM}^{p, q}\left(\widetilde{s}_{1}, \widetilde{s}_{2}, \ldots, \widetilde{s}_{n}\right)=\operatorname{GULHM}^{p, q}\left(\vec{s}_{1}^{\prime}, \widetilde{s}_{2}^{\prime}, \ldots, \widetilde{s}_{n}^{\prime}\right),
$$

where $\widetilde{s}_{i}^{\prime}=\left[s_{\alpha_{i}}^{\prime}, s_{\beta_{i}}^{\prime}\right](i=1,2, \ldots, n)$ is any permutation of $\widetilde{s}_{i}=$ $\left[s_{\alpha_{i}}, s_{\beta_{i}}\right](i=1,2, \ldots, n)$.

Proof. Since $\vec{s}_{i}^{\prime}=\left[s_{\alpha_{i}}^{\prime}, s_{\beta_{i}}^{\prime}\right](i=1,2, \ldots, n)$ is any permutation of $\widetilde{s}_{i}=\left[s_{\alpha_{i}}, s_{\beta_{i}}\right](i=1,2, \ldots, n)$, then

$$
\begin{aligned}
\operatorname{GULHM}^{p, q}\left(\widetilde{s}_{1}, \widetilde{s}_{2}, \ldots, \widetilde{s}_{n}\right) \\
=\left(\frac{2}{n(n+1)} \bigoplus_{i=1, j=i}^{n}\left(\widetilde{s}_{i}^{p} \otimes \widetilde{s}_{j}^{q}\right)\right)^{1 /(p+q)} \\
=\left(\frac{2}{n(n+1)} \bigoplus_{i=1, j=i}^{n}\left(\vec{s}_{i}^{p} \otimes \widetilde{s}_{j}^{q}\right)\right)^{1 /(p+q)} \\
=\operatorname{GULHM}^{p, q}\left(\vec{s}_{1}, \vec{s}_{2}, \ldots, \vec{s}_{n}\right) .
\end{aligned}
$$

Theorem 11 (monotonicity). Let $\widetilde{s}_{i}=\left[s_{\alpha_{i}}, s_{\beta_{i}}\right]$ and $\vec{s}_{i}^{\prime}=$ $\left[s_{\alpha_{i}}^{\prime}, s_{\beta_{i}}^{\prime}\right](i=1,2, \ldots, n)$ be two collections of uncertain linguistic variables. If $s_{\alpha_{i}} \leq s_{\alpha_{i}}^{\prime}, s_{\beta_{i}} \leq s_{\beta_{i}}^{\prime}$ for all $i$, then

$$
\operatorname{GULHM}^{p, q}\left(\widetilde{s}_{1}, \widetilde{s}_{2}, \ldots, \widetilde{s}_{n}\right) \leq \operatorname{GULHM}^{p, q}\left(\widetilde{s}_{1}, \widetilde{s}_{2}^{\prime}, \ldots, \widetilde{s}_{n}^{\prime}\right) .
$$

Proof. Since $s_{\alpha_{i}} \leq s_{\alpha_{i}}^{\prime}, s_{\beta_{i}} \leq s_{\beta_{i}}^{\prime}$ for all $i$, then

$$
\begin{aligned}
& \left(\frac{2}{n(n+1)} \bigoplus_{i=1, j=i}^{n}\left(s_{\alpha_{i}}^{p} \otimes s_{\alpha_{j}}^{q}\right)\right)^{1 /(p+q)} \\
& \leq\left(\frac{2}{n(n+1)} \bigoplus_{i=1, j=i}^{n}\left(s_{\alpha_{i}}^{\prime p} \otimes s_{\alpha_{j}}^{\prime q}\right)\right)^{1 /(p+q)} \\
& \left(\frac{2}{n(n+1)} \bigoplus_{i=1, j=i}^{n}\left(s_{\beta_{i}}^{p} \otimes s_{\beta_{j}}^{q}\right)\right)^{1 /(p+q)} \\
& \leq\left(\frac{2}{n(n+1)} \bigoplus_{i=1, j=i}^{n}\left(s_{\beta_{i}}^{\prime p} \otimes s_{\beta_{j}}^{\prime q}\right)\right)^{1 /(p+q)} .
\end{aligned}
$$

By Definition 2, we get that

$$
\begin{aligned}
& {\left[\left(\frac{2}{n(n+1)} \bigoplus_{i=1, j=i}^{n}\left(s_{\alpha_{i}}^{p} \otimes s_{\alpha_{j}}^{q}\right)\right)^{1 /(p+q)},\right.} \\
& \left.\left[\frac{2}{n(n+1)} \bigoplus_{i=1, j=i}^{n}\left(s_{\beta_{i}}^{p} \otimes s_{\beta_{j}}^{q}\right)\right)^{1 /(p+q)}\right]
\end{aligned}
$$

$$
\begin{aligned}
\leq & {\left[\left(\frac{2}{n(n+1)} \bigoplus_{i=1, j=i}^{n}\left(s_{\alpha_{i}}^{\prime p} \otimes s_{\alpha_{j}}^{\prime q}\right)\right)^{1 /(p+q)},\right.} \\
& \left.\left(\frac{2}{n(n+1)} \bigoplus_{i=1, j=i}^{n}\left(s_{\beta_{i}}^{\prime p} \otimes s_{\beta_{j}}^{\prime q}\right)\right)^{1 /(p+q)}\right] .
\end{aligned}
$$

Thus,

$$
\begin{aligned}
\operatorname{GULHM}^{p, q}\left(\widetilde{s}_{1}, \widetilde{s}_{2}, \ldots, \widetilde{s}_{n}\right) \\
\quad=\left(\frac{2}{n(n+1)} \bigoplus_{i=1, j=i}^{n}\left(\widetilde{s}_{i}^{p} \otimes \widetilde{s}_{j}^{q}\right)\right)^{1 /(p+q)} \\
\quad \leq\left(\frac{2}{n(n+1)} \bigoplus_{i=1, j=i}^{n}\left(\widetilde{s}_{i}^{p} \otimes \widetilde{s}_{j}^{q}\right)\right)^{1 /(p+q)} \\
\quad=\operatorname{GULHM}^{p, q}\left(\vec{s}_{1}, \vec{s}_{2}, \ldots, \widetilde{s}_{n}\right) .
\end{aligned}
$$

Theorem 12 (boundedness). Let $\widetilde{s}_{i}=\left[s_{\alpha_{i}}, s_{\beta_{i}}\right](i=1,2, \ldots$, $n)$ be a collection of uncertain linguistic variables, and

$$
\begin{aligned}
& \widetilde{s}^{-}=\min _{i} \widetilde{s}_{i}=\left[\min _{i} s_{\alpha_{i}}, \min _{i} s_{\beta_{i}}\right], \\
& \widetilde{s}^{+}=\max _{i} \widetilde{s}_{i}=\left[\max _{i} s_{\alpha_{i}}, \max _{i} s_{\beta_{i}}\right] .
\end{aligned}
$$

Then,

$$
\widetilde{s}^{-} \leq \operatorname{GULHM}^{p, q}\left(\widetilde{s}_{1}, \widetilde{s}_{2}, \ldots, \widetilde{s}_{n}\right) \leq \widetilde{s}^{+} .
$$

Proof. Consider the following:

$$
\begin{aligned}
& \operatorname{GULHM}^{p, q}\left(\widetilde{s}_{1}, \widetilde{s}_{2}, \ldots, \widetilde{s}_{n}\right) \\
& =\left[\frac{2}{n(n+1)} \bigoplus_{i=1, j=i}^{n}\left(\widetilde{s}_{i}^{p} \otimes \widetilde{s}_{j}^{q}\right)\right)^{1 /(p+q)} \\
& =\left[\left(\frac{2}{n(n+1)} \bigoplus_{i=1, j=i}^{n}\left(s_{\alpha_{i}}^{p} \otimes s_{\alpha_{j}}^{q}\right)\right)^{1 /(p+q)},\right. \\
& \left.\leq\left[\frac{2}{n(n+1)} \bigoplus_{i=1, j=i}^{n}\left(s_{\beta_{i}}^{p} \otimes s_{\beta_{j}}^{q}\right)\right)^{1 /(p+q)}\right] \\
& =\left[\frac{2}{n(n+1)} \bigoplus_{i=1, j=i}^{n}\left(\left\{\max _{i} s_{\alpha_{i}}\right\}^{p} \otimes\left\{\max _{i} s_{\alpha_{i}}\right\}^{q}\right)\right)^{1 /(p+q)}, \\
& \left.=\left[\max _{i} s_{\alpha_{i}, \max _{i}}^{n} \bigoplus_{\beta_{i}}^{n}\left(\left\{\max _{i}^{+} s_{\beta_{i}}\right\}^{p} \otimes\left\{\max _{i} s_{\beta_{i}}\right\}^{q}\right)\right)^{1 /(p+q)}\right]
\end{aligned}
$$


Similarly, we can prove

$$
\begin{aligned}
\operatorname{GULHM}^{p, q}\left(\widetilde{s}_{1}, \widetilde{s}_{2}, \ldots, \widetilde{s}_{n}\right) \\
=\left(\frac{2}{n(n+1)} \bigoplus_{i=1, j=i}^{n}\left(\widetilde{s}_{i}^{p} \otimes \widetilde{s}_{j}^{q}\right)\right)^{1 /(p+q)} \\
\geq\left[\min _{i} s_{\alpha_{i}}, \min _{i} s_{\beta_{i}}\right]=\widetilde{s}
\end{aligned}
$$

which completes the proof of Theorem 12.

In most cases, the input arguments have their own importance. Each argument should be assigned a weight. Hence, it is necessary to consider the weighted form of the GULHM. In the following, we define the generalized uncertain linguistic weighted Heronian mean (GULWHM).

Definition 13. Let $p, q \geq 0$ and $p, q$ do not take the value 0 simultaneously. Let $\widetilde{s}_{i}=\left[s_{\alpha_{i}}, s_{\beta_{i}}\right](i=1,2, \ldots, n)$ be a collection of uncertain linguistic variables. And $w=$ $\left(w_{1}, w_{2}, \ldots, w_{n}\right)^{T}$ is the weight vector of $\widetilde{s}_{i}=\left[s_{\alpha_{i}}, s_{\beta_{i}}\right](i=$ $1,2, \ldots, n)$, where $w_{i}$ indicates the importance degree of $\widetilde{s}_{i}$, satisfying $w_{i} \geq 0$, and $\sum_{i=1}^{n} w_{i}=1$. If

$$
\begin{aligned}
& \operatorname{GULWHM}_{w}^{p, q}\left(\widetilde{s}_{1}, \widetilde{s}_{2}, \ldots, \widetilde{s}_{n}\right) \\
&=\left(\frac{2}{n(n+1)} \bigoplus_{i=1, j=i}^{n}\left(\left(w_{i} \widetilde{s}_{i}\right)^{p} \otimes\left(w_{j} \widetilde{s}_{j}\right)^{q}\right)\right)^{1 /(p+q)} \\
&= {\left[\left(\frac{2}{n(n+1)} \bigoplus_{i=1, j=i}^{n}\left(\left(w_{i} s_{\alpha_{i}}\right)^{p} \otimes\left(w_{j} s_{\alpha_{j}}\right)^{q}\right)\right)^{1 /(p+q)},\right.} \\
&\left.\left(\frac{2}{n(n+1)} \bigoplus_{i=1, j=i}^{n}\left(\left(w_{i} s_{\beta_{i}}\right)^{p} \otimes\left(w_{j} s_{\beta_{j}}\right)^{q}\right)\right)^{1 /(p+q)}\right],
\end{aligned}
$$

then GULWHM is called the generalized uncertain linguistic weighted Heronian mean (GULWHM). If $p=q=1 / 2$; then the GULWHM reduces to

$$
\begin{aligned}
& \operatorname{GULWHM}_{w}^{1 / 2,1 / 2}\left(\widetilde{s}_{1}, \widetilde{s}_{2}, \ldots, \widetilde{s}_{n}\right) \\
&=\left(\frac{2}{n(n+1)} \bigoplus_{i=1, j=i}^{n}\left(\left(w_{i} \widetilde{s}_{i}\right)^{1 / 2} \otimes\left(w_{j} \widetilde{s}_{j}\right)^{1 / 2}\right)\right) \\
&= {\left[\left(\frac{2}{n(n+1)} \bigoplus_{i=1, j=i}^{n}\left(\left(w_{i} s_{\alpha_{i}}\right)^{1 / 2} \otimes\left(w_{j} s_{\alpha_{j}}\right)^{1 / 2}\right)\right),\right.} \\
&\left.\left(\frac{2}{n(n+1)} \bigoplus_{i=1, j=i}^{n}\left(\left(w_{i} s_{\beta_{i}}\right)^{1 / 2} \otimes\left(w_{j} s_{\beta_{j}}\right)^{1 / 2}\right)\right)\right],
\end{aligned}
$$

which we call the uncertain linguistic weighted Heronian mean (ULWHM).
3.2. The ULGHM and the ULWGHM. The geometric Heronian mean $\left(\mathrm{GHM}_{2}\right)$ proposed by $\mathrm{Yu}$ [33] has the capability to capture the interrelationship among the input arguments. In this section, we will extend the $\mathrm{GHM}_{2}$ to accommodate the situations in which the input arguments are uncertain linguistic variables. Based on the operational rules on uncertain linguistic variables and Definition 6, we give the uncertain linguistic geometric Heronian mean (ULGHM) as follows.

Definition 14. Let $p, q \geq 0$ and $p, q$ do not take the value 0 simultaneously. Let $\widetilde{s}_{i}=\left[s_{\alpha_{i}}, s_{\beta_{i}}\right](i=1,2, \ldots, n)$ be a collection of uncertain linguistic variables. If

$$
\begin{aligned}
& \operatorname{ULGHM}^{p, q}\left(\widetilde{s}_{1}, \widetilde{s}_{2}, \ldots, \widetilde{s}_{n}\right) \\
& =\frac{1}{p+q} \bigotimes_{i=1, j=i}^{n}\left(p \widetilde{s}_{i} \oplus q \widetilde{s}_{j}\right)^{2 / n(n+1)} \\
& =\left[\frac{1}{p+q} \bigotimes_{i=1, j=i}^{n}\left(p s_{\alpha_{i}} \oplus q s_{\alpha_{j}}\right)^{2 / n(n+1)},\right. \\
& \left.\frac{1}{p+q} \bigotimes_{i=1, j=i}^{n}\left(p s_{\beta_{i}} \oplus q s_{\beta_{j}}\right)^{2 / n(n+1)}\right],
\end{aligned}
$$

then the ULGHM is called the uncertain linguistic geometric Heronian mean (ULGHM). If $p=q$, then the ULGHM reduces to

$$
\begin{aligned}
& \operatorname{ULGHM}^{p, q}\left(\widetilde{s}_{1}, \widetilde{s}_{2}, \ldots, \widetilde{s}_{n}\right) \\
& =\frac{1}{2} \bigotimes_{i=1, j=i}^{n}\left(\widetilde{s}_{i} \oplus \widetilde{s}_{j}\right)^{2 / n(n+1)} \\
& =\frac{1}{2}\left[\bigotimes_{i=1, j=i}^{n}\left(s_{\alpha_{i}} \oplus s_{\alpha_{j}}\right)^{2 / n(n+1)}, \bigotimes_{i=1, j=i}^{n}\left(s_{\beta_{i}} \oplus s_{\beta_{j}}\right)^{2 / n(n+1)}\right]
\end{aligned}
$$

which we call the uncertain linguistic evolution Heronian mean (ULEHM).

In the following, we investigate the desirable properties of the ULGHM, and they can be derived easily.

Theorem 15 (idempotency). Let $\widetilde{s}_{i}=\left[s_{\alpha_{i}}, s_{\beta_{i}}\right](i=1,2, \ldots$, $n)$ be a collection of uncertain linguistic variables. If all $\widetilde{s}_{i}$ are equal, that is, $\widetilde{s}_{i}=\widetilde{s}=\left[s_{\alpha}, s_{\beta}\right]$ for all $i$, then

$$
U L G H M^{p, q}\left(\widetilde{s}_{1}, \widetilde{s}_{2}, \ldots, \widetilde{s}_{n}\right)=\widetilde{s} .
$$

Theorem 16 (permutation). Let $\widetilde{s}_{i}=\left[s_{\alpha_{i}}, s_{\beta_{i}}\right]$ and $\widetilde{s}_{i}^{\prime}=$ $\left[s_{\alpha_{i}}^{\prime}, s_{\beta_{i}}^{\prime}\right](i=1,2, \ldots, n)$ be two collections of uncertain linguistic variables; then

$$
\operatorname{ULGHM}^{p, q}\left(\widetilde{s}_{1}, \widetilde{s}_{2}, \ldots, \widetilde{s}_{n}\right)=U L G H M^{p, q}\left(\widetilde{s}_{1}, \widetilde{s}_{2}, \ldots, \widetilde{s}_{n}^{\prime}\right),
$$

where $\vec{s}_{i}^{\prime}=\left[s_{\alpha_{i}}^{\prime}, s_{\beta_{i}}^{\prime}\right](i=1,2, \ldots, n)$ is any permutation of $\widetilde{s}_{i}=$ $\left[s_{\alpha_{i}}, s_{\beta_{i}}\right](i=1,2, \ldots, n)$. 
Theorem 17 (monotonicity). Let $\widetilde{s}_{i}=\left[s_{\alpha_{i}}, s_{\beta_{i}}\right]$ and $\vec{s}_{i}^{\prime}=$ $\left[s_{\alpha_{i}}^{\prime}, s_{\beta_{i}}^{\prime}\right](i=1,2, \ldots, n)$ be two collections of uncertain linguistic variables. If $s_{\alpha_{i}} \leq s_{\alpha_{i}}^{\prime}, s_{\beta_{i}} \leq s_{\beta_{i}}^{\prime}$ for all $i$, then

$$
U L G H M^{p, q}\left(\widetilde{s}_{1}, \widetilde{s}_{2}, \ldots, \widetilde{s}_{n}\right) \leq U L G H M^{p, q}\left(\widetilde{s}_{1}^{\prime}, \widetilde{s}_{2}, \ldots, \widetilde{s}_{n}^{\prime}\right) .
$$

Theorem 18 (boundedness). Let $\widetilde{s}_{i}=\left[s_{\alpha_{i}}, s_{\beta_{i}}\right](i=1,2, \ldots$, $n)$ be a collection of uncertain linguistic variables, and

$$
\begin{aligned}
& \widetilde{s}^{-}=\min _{i} \widetilde{s}_{i}=\left[\min _{i} s_{\alpha_{i}}, \min _{i} s_{\beta_{i}}\right], \\
& \widetilde{s}^{+}=\max _{i} \widetilde{s}_{i}=\left[\max _{i} s_{\alpha_{i}}, \max _{i} s_{\beta_{i}}\right],
\end{aligned}
$$

then

$$
\widetilde{s}^{-} \leq \operatorname{ULGHM} M^{p, q}\left(\widetilde{s}_{1}, \widetilde{s}_{2}, \ldots, \widetilde{s}_{n}\right) \leq \widetilde{s}^{+}
$$

It is noted that the uncertain linguistic geometric Heronian mean (ULGHM) does not consider the importance of each argument. In the following, we introduce the uncertain linguistic weighted geometric Heronian mean (ULWGHM).

Definition 19. Let $p, q \geq 0$ and $p, q$ do not take the value 0 simultaneously. Let $\widetilde{s}_{i}=\left[s_{\alpha_{i}}, s_{\beta_{i}}\right](i=1,2, \ldots, n)$ be a collection of uncertain linguistic variables. If

$$
\begin{aligned}
& \operatorname{ULWGHM}_{w}^{p, q}\left(a_{1}, a_{2}, \ldots, a_{n}\right) \\
& =\frac{1}{p+q} \bigotimes_{i=1, j=i}^{n}\left(p \widetilde{s}_{i}^{w_{i}} \oplus q \widetilde{s}_{j}^{w_{j}}\right)^{2 / n(n+1)} \\
& =\left[\frac{1}{p+q} \bigotimes_{i=1, j=i}^{n}\left(p s_{\alpha_{i}}^{w_{i}} \oplus q s_{\alpha_{j}}^{w_{j}}\right)^{2 / n(n+1)},\right. \\
& \left.\frac{1}{p+q} \bigotimes_{i=1, j=i}^{n}\left(p s_{\beta_{i}}^{w_{i}} \oplus q s_{\beta_{j}}^{w_{j}}\right)^{2 / n(n+1)}\right],
\end{aligned}
$$

then ULWGHM is called the uncertain linguistic weighted geometric Heronian mean (ULWGHM). If $p=q$, then the ULWGHM reduces to

$$
\begin{aligned}
& \operatorname{ULWGHM}_{w}^{p, q}\left(a_{1}, a_{2}, \ldots, a_{n}\right) \\
& =\frac{1}{2} \bigotimes_{i=1, j=i}^{n}\left(\widetilde{s}_{i}^{w_{i}} \oplus \widetilde{s}_{j}^{w_{j}}\right)^{2 / n(n+1)} \\
& =\frac{1}{2}\left[\bigotimes_{i=1, j=i}^{n}\left(s_{\alpha_{i}}^{w_{i}} \oplus s_{\alpha_{j}}^{w_{j}}\right)^{2 / n(n+1)}, \bigotimes_{i=1, j=i}^{n}\left(s_{\beta_{i}}^{w_{i}} \oplus s_{\beta_{j}}^{w_{j}}\right)^{2 / n(n+1)}\right]
\end{aligned}
$$

which we call the uncertain linguistic weighted evolution Heronian mean (ULWGHM).

\section{A Method for Multiple Attribute Decision Making Based on Heronian Means under Uncertain Linguistic Environment}

In this section, we consider a multiple attribute decision making problem with uncertain linguistic information. The generalized uncertain linguistic weighted Heronian mean (GULWHM) or the uncertain linguistic weighted geometric Heronian mean (ULWGHM) proposed in Section 3 will be used to solve the multiple attribute decision making problem.

Let $A=\left\{A_{1}, A_{2}, \ldots, A_{m}\right\}$ be the set of alternatives and $C=\left\{C_{1}, C_{2}, \ldots, C_{n}\right\}$ the set of attributes, whose weight vector is $w=\left(w_{1}, w_{2}, \ldots, w_{n}\right)^{T}$ such that $w_{j} \in[0,1], \sum_{j=1}^{n} w_{j}=$ 1. The decision makers use the uncertain linguistic variable to provide the linguistic expression under the attribute $C_{j}$ for the alternative $A_{i}$ and construct the uncertain linguistic decision matrix $D=\left(\tilde{d}_{i j}\right)_{m \times n}$. In the following, based on the GULWHM or the ULWGHM, we develop an approach to multiple attribute decision making with uncertain linguistic information.

Step 1. Utilize the GULWHM as

$$
\begin{aligned}
& \widetilde{d}_{i}=\left[s_{\alpha_{i}}, s_{\beta_{i}}\right]=\operatorname{GULWHM}_{w}^{p, q}\left(\widetilde{d}_{i 1}, \widetilde{d}_{i 2}, \ldots, \widetilde{d}_{i n}\right)
\end{aligned}
$$

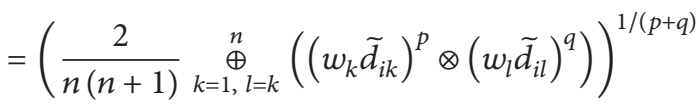

$$
\begin{aligned}
& (i=1,2, \ldots, m) \text {, }
\end{aligned}
$$

or the ULWGHM as

$$
\begin{aligned}
& \widetilde{d}_{i}=\left[s_{\alpha_{i}}, s_{\beta_{i}}\right]=\operatorname{ULWGHM}_{w}^{p, q}\left(\widetilde{d}_{i 1}, \widetilde{d}_{i 2}, \ldots, \widetilde{d}_{i n}\right) \\
& =\frac{1}{p+q} \stackrel{\otimes}{\otimes}_{k=1, l=k}^{n}\left(p \widetilde{d}_{i k}^{w_{k}} \oplus q \widetilde{d}_{i l}^{w_{l}}\right)^{2 / n(n+1)} \quad(i=1,2, \ldots, m)
\end{aligned}
$$

to get the overall attribute value $\tilde{d}_{i}$ of the alternative $A_{i}(i=$ $1,2, \ldots, m)$.

Step 2. To rank these overall attribute values $\widetilde{d}_{i}(i=1,2, \ldots$, $m)$, we first compare each $\tilde{d}_{i}$ with all the $\tilde{d}_{j}(j=1,2, \ldots, m)$ by using (2). Then a complementary matrix $P=\left(p_{i j}\right)_{m \times m}$ is developed, where

$$
\begin{array}{r}
p_{i j}=p\left(\tilde{d}_{i} \geq \tilde{d}_{j}\right), \quad p_{i j} \geq 0, p_{i j}+p_{j i}=1, \\
p_{i i}=0.5, \quad i, j=1,2, \ldots, m .
\end{array}
$$

Summing all the elements in each line of matrix $P=$ $\left(p_{i j}\right)_{m \times m}$, we have $p_{i}=\sum_{j=1}^{m} p_{i j}, \quad i=1,2, \ldots, m$. Then we rank the overall attribute values $\tilde{d}_{i}$ in descending order according to the values of $p_{i}(i=1,2, \ldots, m)$.

Step 3. Rank all the alternatives $A_{i}$ and select the desirable one in accordance with the values of $\widetilde{d}_{i}(i=1,2, \ldots, m)$.

Step 4. End. 
TABLE 1: Uncertain linguistic decision matrix $D$.

\begin{tabular}{lcccc}
\hline & $C_{1}$ & $C_{2}$ & $C_{3}$ & $C_{4}$ \\
\hline$A_{1}$ & {$\left[s_{3}, s_{4}\right]$} & {$\left[s_{5}, s_{7}\right]$} & {$\left[s_{2}, s_{3}\right]$} & {$\left[s_{3}, s_{4}\right]$} \\
$A_{2}$ & {$\left[s_{2}, s_{3}\right]$} & {$\left[s_{2}, s_{3}\right]$} & {$\left[s_{4}, s_{6}\right]$} & {$\left[s_{4}, s_{5}\right]$} \\
$A_{3}$ & {$\left[s_{4}, s_{5}\right]$} & {$\left[s_{4}, s_{6}\right]$} & {$\left[s_{5}, s_{6}\right]$} & {$\left[s_{6}, s_{7}\right]$} \\
$A_{4}$ & {$\left[s_{3}, s_{5}\right]$} & {$\left[s_{6}, s_{7}\right]$} & {$\left[s_{4}, s_{5}\right]$} & {$\left[s_{5}, s_{6}\right]$} \\
\hline
\end{tabular}

\section{Example Illustration and Discussion}

In this section, an example adapted from [29] is given to illustrate the application of the methods proposed in this paper.

\subsection{Example Illustration}

Example 20 (see [29]). Suppose an organization plans to implement ERP system. The first step is to form a project team that consists of $\mathrm{CIO}$ and two senior representatives from user departments. By collecting all possible information about ERP vendors and systems, project team chooses four potential ERP systems $A_{i}(i=1,2,3,4)$ as candidates. The company employs some external professional organizations (or experts) to aid this decision making. The project team selects four attributes to evaluate the alternatives: (1) function and technology $C_{1}$, (2) strategic fitness $C_{2}$, (3) vendor's ability $C_{3}$, and (4) vendor's reputation $C_{4}$. Decision makers use the uncertain linguistic variables to evaluate the four possible alternatives $A_{i}(i=1,2,3,4)$ under the above four attributes (whose weight vector is $w=(0.2,0.1,0.3,0.4)^{T}$ ) and construct the uncertain linguistic decision matrix $D=$ $\left(\tilde{d}_{i j}\right)_{4 \times 4}$ listed in Table 1.

In the following, we use the proposed methods to get the most desirable system.

Step 1. Utilize the GULWHM as

$$
\begin{array}{r}
\tilde{d}_{i}=\left[s_{\alpha_{i}}, s_{\beta_{i}}\right]=\operatorname{GULWHM}_{w}^{p, q}\left(\tilde{d}_{i 1}, \tilde{d}_{i 2}, \ldots, \tilde{d}_{i n}\right) \\
=\left(\frac{2}{n(n+1)} \bigoplus_{k=1, l=k}^{n}\left(\left(w_{k} \tilde{d}_{i k}\right)^{p} \otimes\left(w_{l} \tilde{d}_{i l}\right)^{q}\right)\right)^{1 /(p+q)} \\
(i=1,2, \ldots, m)
\end{array}
$$

to obtain the overall attribute value $\tilde{d}_{i}$ for the alternative $A_{i}(i=1,2,3,4)$, and let $p=q=1$. We have

$$
\begin{array}{ll}
\widetilde{d}_{1}=\left[s_{0.74}, s_{1.01}\right], & \widetilde{d}_{2}=\left[s_{0.89}, s_{1.22}\right], \\
\widetilde{d}_{3}=\left[s_{1.32}, s_{1.59}\right], & \widetilde{d}_{4}=\left[s_{1.13}, s_{1.43}\right] .
\end{array}
$$

Step 2. To rank these overall attribute values $\widetilde{d}_{i}(i=1, \ldots, 4)$, we first compare each $\widetilde{d}_{i}$ with all the $\widetilde{d}_{j}(j=1, \ldots, 4)$ by using (2). Then a complementary matrix $P=\left(p_{i j}\right)_{4 \times 4}$ is developed as

$$
P=\left(p_{i j}\right)_{4 \times 4}=\left[\begin{array}{llll}
0.500 & 0.200 & 0.000 & 0.000 \\
0.800 & 0.500 & 0.000 & 0.143 \\
1.000 & 1.000 & 0.500 & 0.807 \\
1.000 & 0.857 & 0.193 & 0.500
\end{array}\right]
$$

Summing all the elements in each line of matrix $P=$ $\left(p_{i j}\right)_{4 \times 4}$, we have

$$
\begin{array}{ll}
p_{1}=0.700, & p_{2}=1.443, \\
p_{3}=3.307, & p_{4}=2.550 .
\end{array}
$$

Then we rank the overall attribute values $\widetilde{d}_{i}$ in descending order according to the values of $p_{i}(i=1,2,3,4)$ as

$$
\tilde{d}_{3}>\tilde{d}_{4}>\tilde{d}_{2}>\tilde{d}_{1} \text {. }
$$

Step 3. Rank all the alternatives $A_{i}$ in accordance with the values of $\tilde{d}_{i}(i=1,2,3,4)$ as

$$
A_{3}>A_{4}>A_{2}>A_{1} \text {. }
$$

Thus, the most desirable system is $A_{3}$.

If we use the ULWGHM to solve the above multiple attribute decision making problem and let $p=q$, then the overall attribute values $\widetilde{d}_{i}$ of the alternative $A_{i}(i=1,2,3,4)$ can be obtained as follows:

$$
\begin{array}{ll}
\widetilde{d}_{1}=\left[s_{1.30}, s_{1.41}\right], & \widetilde{d}_{2}=\left[s_{1.35}, s_{1.47}\right], \\
\widetilde{d}_{3}=\left[s_{1.51}, s_{1.59}\right], & \widetilde{d}_{4}=\left[s_{1.45}, s_{1.55}\right] .
\end{array}
$$

To rank these overall attribute values $\widetilde{d}_{i}(i=1,2,3,4)$, we first compare each $\tilde{d}_{i}$ with all the $\widetilde{d}_{j}(j=1,2,3,4)$ by using (2). Then a complementary matrix $P=\left(p_{i j}\right)_{4 \times 4}$ is developed as

$$
P=\left(p_{i j}\right)_{4 \times 4}=\left[\begin{array}{llll}
0.500 & 0.261 & 0.000 & 0.000 \\
0.739 & 0.500 & 0.000 & 0.091 \\
1.000 & 1.000 & 0.500 & 0.778 \\
1.000 & 0.909 & 0.222 & 0.500
\end{array}\right]
$$

Summing all the elements in each line of matrix $P=$ $\left(p_{i j}\right)_{4 \times 4}$, we have

$$
\begin{array}{ll}
p_{1}=0.761, & p_{2}=1.330 \\
p_{3}=3.278, & p_{4}=2.631 .
\end{array}
$$

Then we rank the overall attribute values $\widetilde{d}_{i}$ in descending order according to the values of $p_{i}(i=1,2,3,4)$ as

$$
\tilde{d}_{3}>\tilde{d}_{4}>\tilde{d}_{2}>\tilde{d}_{1} .
$$

Rank all the alternatives $A_{i}$ in accordance with the values of $\widetilde{d}_{i}(i=1,2,3,4)$ as

$$
A_{3}>A_{4}>A_{2}>A_{1} .
$$

Thus, the most desirable system is $A_{3}$ and the ranking is the same as obtained by the GULWHM. 
TABLE 2: Overall attribute values by the GULWHM${ }_{w}^{p, p}$ and the rankings of the alternatives.

\begin{tabular}{|c|c|c|c|c|c|}
\hline & $A_{1}$ & $A_{2}$ & $A_{3}$ & $A_{4}$ & Ranking \\
\hline $\operatorname{GULWHM}_{w}^{1 / 2,1 / 2}$ & {$\left[s_{0.71}, s_{0.98}\right]$} & {$\left[s_{0.76}, s_{1.07}\right]$} & {$\left[s_{1.18}, s_{1.46}\right]$} & {$\left[s_{1.04}, s_{1.34}\right]$} & $A_{3}>A_{4}>A_{2}>A_{1}$ \\
\hline GULWHM $_{w}^{1,1}$ & {$\left[s_{0.74}, s_{1.01}\right]$} & {$\left[s_{0.89}, s_{1.22}\right]$} & {$\left[s_{1.32}, s_{1.59}\right]$} & {$\left[s_{1.13}, s_{1.43}\right]$} & $A_{3}>A_{4}>A_{2}>A_{1}$ \\
\hline GULWHM $_{w}^{2,2}$ & {$\left[s_{0.80}, s_{1.09}\right]$} & {$\left[s_{1.07}, s_{1.44}\right]$} & {$\left[s_{1.55}, s_{1.84}\right]$} & {$\left[s_{1.30}, s_{1.60}\right]$} & $A_{3}>A_{4}>A_{2}>A_{1}$ \\
\hline GULWHM $_{w}^{5,5}$ & {$\left[s_{0.96}, s_{1.28}\right]$} & {$\left[s_{1.30}, s_{1.70}\right]$} & {$\left[s_{1.93}, s_{2.25}\right]$} & {$\left[s_{1.60}, s_{1.79}\right]$} & $A_{3}>A_{4}>A_{2}>A_{1}$ \\
\hline GULWHM $_{w}^{10,10}$ & {$\left[s_{1.07}, s_{1.43}\right]$} & {$\left[s_{1.43}, s_{1.82}\right]$} & {$\left[s_{2.14}, s_{2.50}\right]$} & {$\left[s_{1.78}, s_{2.14}\right]$} & $A_{3}>A_{4}>A_{2}>A_{1}$ \\
\hline
\end{tabular}

TABLE 3: Overall attribute values by the GULWHM ${ }_{w}^{1, q}$ and the rankings of the alternatives.

\begin{tabular}{lccccc}
\hline & $A_{1}$ & $A_{2}$ & $A_{3}$ & $A_{4}$ & Ranking \\
\hline GULWHM $_{w}^{1,1 / 2}$ & {$\left[s_{0.69}, s_{0.95}\right]$} & {$\left[s_{0.77}, s_{1.08}\right]$} & {$\left[s_{1.17}, s_{1.43}\right]$} & {$\left[s_{1.02}, s_{1.31}\right]$} & $A_{3}>A_{4}>A_{2}>A_{1}$ \\
GULWHM $_{w}^{1,1}$ & {$\left[s_{0.74}, s_{1.01}\right]$} & {$\left[s_{0.89}, s_{1.22}\right]$} & {$\left[s_{1.32}, s_{1.59}\right]$} & {$\left[s_{1.13}, s_{1.43}\right]$} & $A_{3}>A_{4}>A_{2}>A_{1}$ \\
GULWHM $_{w}^{1,2}$ & {$\left[s_{0.81}, s_{1.10}\right]$} & {$\left[s_{1.04}, s_{1.41}\right]$} & {$\left[s_{1.53}, s_{1.83}\right]$} & {$\left[s_{1.29}, s_{1.60}\right]$} & $A_{3}>A_{4}>A_{2}>A_{1}$ \\
GULWHM $_{w}^{1,5}$ & {$\left[s_{0.95}, s_{1.28}\right]$} & {$\left[s_{1.26}, s_{1.65}\right]$} & {$\left[s_{1.87}, s_{2.19}\right]$} & {$\left[s_{1.56}, s_{1.90}\right]$} & $A_{3}>A_{4}>A_{2}>A_{1}$ \\
GULWHM $_{w}^{1,10}$ & {$\left[s_{1.05}, s_{1.41}\right]$} & {$\left[s_{1.39}, s_{1.78}\right]$} & {$\left[s_{2.09}, s_{2.44}\right]$} & {$\left[s_{1.74}, s_{2.10}\right]$} & $A_{3}>A_{4}>A_{2}>A_{1}$ \\
\hline
\end{tabular}

TABLE 4: Overall attribute values by the GULWHM ${ }_{w}^{p, 1}$ and the rankings of the alternatives.

\begin{tabular}{lccccc}
\hline & $A_{1}$ & $A_{2}$ & $A_{3}$ & $A_{4}$ & Ranking \\
\hline GULWHM $_{w}^{1 / 2,1}$ & {$\left[s_{0.76}, s_{1.04}\right]$} & {$\left[s_{0.90}, s_{1.24}\right]$} & {$\left[s_{1.36}, s_{1.64}\right]$} & {$\left[s_{1.17}, s_{1.48}\right]$} & $A_{3}>A_{4}>A_{2}>A_{1}$ \\
GULWHM $_{w}^{1,1}$ & {$\left[s_{0.74}, s_{1.01}\right]$} & {$\left[s_{0.89}, s_{1.22}\right]$} & {$\left[s_{1.32}, s_{1.59}\right]$} & {$\left[s_{1.13}, s_{1.43}\right]$} & $A_{3}>A_{4}>A_{2}>A_{1}$ \\
GULWHM $_{w}^{2,1}$ & {$\left[s_{0.74}, s_{1.02}\right]$} & {$\left[s_{0.96}, s_{1.31}\right]$} & {$\left[s_{1.38}, s_{1.65}\right]$} & {$\left[s_{1.16}, s_{1.45}\right]$} & $A_{3}>A_{4}>A_{2}>A_{1}$ \\
GULWHM $_{w}^{5,1}$ & {$\left[s_{0.84}, s_{1.12}\right]$} & {$\left[s_{1.16}, s_{1.55}\right]$} & {$\left[s_{1.68}, s_{1.96}\right]$} & {$\left[s_{1.39}, s_{1.68}\right]$} & $A_{3}>A_{4}>A_{2}>A_{1}$ \\
GULWHM $_{w}^{10,1}$ & {$\left[s_{0.97}, s_{1.30}\right]$} & {$\left[s_{1.31}, s_{1.70}\right]$} & {$\left[s_{1.95}, s_{2.28}\right]$} & {$\left[s_{1.62}, s_{1.95}\right]$} & $A_{3}>A_{4}>A_{2}>A_{1}$ \\
\hline
\end{tabular}

TABLE 5: Overall attribute values by the $\mathrm{ULWGHM}_{w}^{1, q}$ and the rankings of the alternatives.

\begin{tabular}{|c|c|c|c|c|c|}
\hline & $A_{1}$ & $A_{2}$ & $A_{3}$ & $A_{4}$ & Ranking \\
\hline ULWGHM $_{w}^{1,1 / 2}$ & {$\left[s_{1.28}, s_{1.39}\right]$} & {$\left[s_{1.32}, s_{1.43}\right]$} & {$\left[s_{1.47}, s_{1.57}\right]$} & {$\left[s_{1.41}, s_{1.51}\right]$} & $A_{3}>A_{4}>A_{2}>A_{1}$ \\
\hline $\mathrm{ULWGHM}_{w}^{w, 1}$ & {$\left[s_{1.30}, s_{1.41}\right]$} & {$\left[s_{1.35}, s_{1.47}\right]$} & {$\left[s_{1.51}, s_{1.59}\right]$} & {$\left[s_{1.45}, s_{1.55}\right]$} & $A_{3}>A_{4}>A_{2}>A_{1}$ \\
\hline $\mathrm{ULWGHM}_{w}^{1,2}$ & {$\left[s_{1.31}, s_{1.43}\right]$} & {$\left[s_{1.39}, s_{1.51}\right]$} & {$\left[s_{1.55}, s_{1.64}\right]$} & {$\left[s_{1.49}, s_{1.58}\right]$} & $A_{3}>A_{4}>A_{2}>A_{1}$ \\
\hline $\mathrm{ULWGHM}_{w}^{1,5}$ & {$\left[s_{1.33}, s_{1.45}\right]$} & {$\left[s_{1.42}, s_{1.55}\right]$} & {$\left[s_{1.59}, s_{1.68}\right]$} & {$\left[s_{1.52}, s_{1.62}\right]$} & $A_{3}>A_{4}>A_{2}>A_{1}$ \\
\hline ULWGHM $_{w}^{1,10}$ & {$\left[s_{1.34}, s_{1.46}\right]$} & {$\left[s_{1.44}, s_{1.57}\right]$} & {$\left[s_{1.61}, s_{1.70}\right]$} & {$\left[s_{1.53}, s_{1.64}\right]$} & $A_{3}>A_{4}>A_{2}>A_{1}$ \\
\hline
\end{tabular}

5.2. Discussion. If the parameter $p$ or $q$ takes the value of zero, then the GULWHM and ULWGHM cannot capture the interrelationship of the input arguments. Moreover, different overall attribute values $\widetilde{d}_{i}$ of the alternatives $A_{i}(i=$ $1,2,3,4)$ can be obtained, and it needs much more calculation effort as the parameters $p$ and $q$ change. Here, we will list some of them. From Table 2, we can find that the overall attribute values obtained by the GULWHM become bigger as the parameters $p$ and $q$ increase simultaneously for the same aggregation arguments. If the parameter $p$ is fixed (without loss of generality, $p$ takes the value 1 ) and the parameter $q$ increases, the overall attribute values obtained by the GULWHM and shown in Table 3 become bigger for the same aggregation arguments. Similarly, if the parameter $q$ is fixed $(q=1)$, the aggregated results in Table 4 show that the overall attribute values obtained by the GULWHM for the same aggregation arguments firstly experience a decrease and then become bigger as the parameter $p$ increases. The different parameters play an important part in decision making. The decision makers who take a pessimistic view for prospect can choose the smaller values of the parameters $p$ and $q$, while the decision makers who take an optimistic view for prospect can choose the bigger values of the parameters $p$ or $q$.

If we utilize the ULWGHM to aggregate the arguments, some different overall attribute values $\widetilde{d}_{i}$ of the alternatives $A_{i}(i=1,2,3,4)$ are listed in Tables 5 and 6 . If the parameter $p$ is fixed $(p=1)$, the overall attribute values obtained by the ULWGHM become bigger as the parameter $q$ increases for the same aggregation arguments. If the parameter $q$ is fixed $(q=1)$, the overall attribute values obtained by the ULWGHM become smaller as the parameter $p$ increases for the same aggregation arguments. Therefore, the decision makers who take a pessimistic view for prospect can choose the smaller values of the parameter $q$ or the bigger values of the parameter $p$, while the decision makers who take an optimistic view for prospect can choose the bigger values of the parameter $q$ or the smaller values of the parameter $p$. From Tables 2 to 6 , we can find that the overall attribute values 
TABLE 6: Overall attribute values by the $\mathrm{ULWGHM}_{w}^{p, 1}$ and the rankings of the alternatives.

\begin{tabular}{|c|c|c|c|c|c|}
\hline & $A_{1}$ & $A_{2}$ & $A_{3}$ & $A_{4}$ & Ranking \\
\hline $\mathrm{ULWGHM}_{w}^{1 / 2,1}$ & {$\left[s_{1.31}, s_{1.43}\right]$} & {$\left[s_{1.39}, s_{1.51}\right]$} & {$\left[s_{1.55}, s_{1.64}\right]$} & {$\left[s_{1.49}, s_{1.58}\right]$} & $A_{3}>A_{4}>A_{2}>A_{1}$ \\
\hline $\mathrm{ULWGHM}_{w}^{1,1}$ & {$\left[s_{1.30}, s_{1.41}\right]$} & {$\left[s_{1.35}, s_{1.47}\right]$} & {$\left[s_{1.51}, s_{1.59}\right]$} & {$\left[s_{1.45}, s_{1.55}\right]$} & $A_{3}>A_{4}>A_{2}>A_{1}$ \\
\hline $\mathrm{ULWGHM}_{w}^{2,1}$ & {$\left[s_{1.28}, s_{1.39}\right]$} & {$\left[s_{1.32}, s_{1.43}\right]$} & {$\left[s_{1.47}, s_{1.54}\right]$} & {$\left[s_{1.41}, s_{1.51}\right]$} & $A_{3}>A_{4}>A_{2}>A_{1}$ \\
\hline ULWGHM $_{w}^{5,1}$ & {$\left[s_{1.26}, s_{1.36}\right]$} & {$\left[s_{1.28}, s_{1.39}\right]$} & {$\left[s_{1.43}, s_{1.50}\right]$} & {$\left[s_{1.38}, s_{1.47}\right]$} & $A_{3}>A_{4}>A_{2}>A_{1}$ \\
\hline ULWGHM $_{w}^{10,1}$ & {$\left[s_{1.25}, s_{1.35}\right]$} & {$\left[s_{1.26}, s_{1.37}\right]$} & {$\left[s_{1.40}, s_{1.47}\right]$} & {$\left[s_{1.36}, s_{1.45}\right]$} & $A_{3}>A_{4}>A_{2}>A_{1}$ \\
\hline
\end{tabular}

of each alternative derived by the GULWHM or ULWGHM depend on the choice of the parameters $p$ and $q$, but the ranking is kept unchanged.

\section{Concluding Remarks}

The Heronian mean can reflect the correlation of the aggregated arguments and is usually used to aggregate the information taken the form of numerical numbers. In this paper, we extend the Heronian mean to accommodate the situation where the input arguments are uncertain linguistic variables and develop some uncertain linguistic Heronian means such as the generalized uncertain linguistic Heronian mean (GULHM) and uncertain linguistic geometric Heronian mean (ULGHM). Some desirable properties of these means such as idempotency, permutation, monotonicity, and boundedness are also discussed. Moreover, to aggregate uncertain linguistic variables and embody different importance of the input arguments, we then define the generalized uncertain linguistic weighted Heronian mean (GULWHM) and uncertain linguistic weighted geometric Heronian mean (ULWGHM). The proposed means take the interrelationship of the input arguments into account, and it is a flexible multiple attribute decision making method in that the decision makers can choose different values of the parameters $p$ and $q$ according to their actual needs. To demonstrate the effectiveness and feasibility of the developed uncertain linguistic Heronian means, an example about ERP system is given. In future research, we will continue to study the Heronian mean, and some other types of Heronian mean will also be investigated.

\section{Acknowledgments}

This work was supported by the National Natural Science Foundation of China (no. 71171112), the Key Project of University Philosophy and Social Science in Jiangsu Province (no. 2012ZDIXM007 and 2012JDXM003), the Funding of Jiangsu Innovation Program for Graduate Education (no. CXLX13_171), the Fundamental Research Funds for the Central Universities, and the Young Teachers' Science Foundation of Anhui University of Technology (no. QZ201321).

\section{References}

[1] F. Herrera and E. Herrera-Viedma, "Linguistic decision analysis: steps for solving decision problems under linguistic information," Fuzzy Sets and Systems, vol. 115, no. 1, pp. 67-82, 2000.
[2] F. Herrera and L. Martinez, "A 2-tuple fuzzy linguistic representation model for computing with words," IEEE Transactions on Fuzzy Systems, vol. 8, no. 6, pp. 746-752, 2000.

[3] F. Herrera and L. Martínez, "A model based on linguistic 2tuples for dealing with multigranular hierarchical linguistic contexts in multi-expert decision-making," IEEE Transactions on Systems, Man, and Cybernetics B, vol. 31, no. 2, pp. 227-234, 2001.

[4] F. Herrera, E. Herrera-Viedma, and J. L. Verdegay, "A rational consensus model in group decision making using linguistic assessments," Fuzzy Sets and Systems, vol. 88, no. 1, pp. 31-49, 1997.

[5] F. Herrera, E. Herrera-Viedma, and J. L. Verdegay, "Direct approach processes in group decision making using linguistic OWA operators," Fuzzy Sets and Systems, vol. 79, no. 2, pp. 175190, 1996.

[6] F. Herrera and E. Herrera-Viedma, "Aggregation operators for linguistic weighted information," IEEE Transactions on Systems, Man, and Cybernetics A, vol. 27, no. 5, pp. 646-656, 1997.

[7] F. Herrera, E. Herrera-Viedma, and J. L. Verdegay, "A model of consensus in group decision making under linguistic assessments," Fuzzy Sets and Systems, vol. 78, no. 1, pp. 73-87, 1996.

[8] G. Bordogna, M. Fedrizzi, and G. Pasi, "A linguistic modeling of consensus in group decision making based on OWA operators," IEEE Transactions on Systems, Man, and Cybernetics A, vol. 27, no. 1, pp. 126-132, 1997.

[9] Z. B. Wu and Y. H. Chen, "The maximizing deviation method for group multiple attribute decision making under linguistic environment," Fuzzy Sets and Systems, vol. 158, no. 14, pp. 16081617, 2007.

[10] J. J. Zhu and K. W. Hipel, "Multiple stages grey target decision making method with incomplete weight based on multigranularity linguistic label," Information Sciences, vol. 212, no. 12, pp. 15-32, 2012.

[11] J. H. Park, M. G. Gwak, and Y. C. Kwun, "Linguistic harmonic mean operators and their applications to group decision making," International Journal of Advanced Manufacturing Technology, vol. 57, no. 1-4, pp. 411-419, 2011.

[12] Z. S. Xu, "Deviation measures of linguistic preference relations in group decision making," Omega, vol. 33, no. 3, pp. 249-254, 2005.

[13] R. R. Yager, "On ordered weighted averaging aggregation operators in multicriteria decisionmaking," IEEE Transactions on Systems, Man, and Cybernetics, vol. 212, no. 12, pp. 183-190, 1988.

[14] Z. S. Xu, Uncertain Multiple Attribute Decision Making: Methods and Applications, Tsinghua University Press, Beijing, China, 2004.

[15] Z. S. Xu, "A note on linguistic hybrid arithmetic averaging operator in multiple attribute group decision making with 
linguistic information," Group Decision and Negotiation, vol. 15, no. 6, pp. 593-604, 2006.

[16] Z. S. Xu, "EOWA and EOWG operators for aggregating linguistic labels based on linguistic preference relations," International Journal of Uncertainty, Fuzziness and Knowledge-Based Systems, vol. 12, no. 6, pp. 791-810, 2004.

[17] Z. S. Xu, "A method based on linguistic aggregation operators for group decision making with linguistic preference relations," Information Sciences, vol. 166, no. 1-4, pp. 19-30, 2004.

[18] Z. Xu, "Uncertain linguistic aggregation operators based approach to multiple attribute group decision making under uncertain linguistic environment," Information Sciences, vol. 168, no. 1-4, pp. 171-184, 2004.

[19] Z. S. Xu, "An approach to pure linguistic multiple attribute decision making under uncertainty," International Journal of Information Technology and Decision Making, vol. 4, no. 2, pp. 197-206, 2005.

[20] Z. S. Xu, "An approach based on the uncertain LOWG and induced uncertain LOWG operators to group decision making with uncertain multiplicative linguistic preference relations," Decision Support Systems, vol. 41, no. 2, pp. 488-499, 2006.

[21] G.-W. Wei, "Uncertain linguistic hybrid geometric mean operator and its application to group decision making under uncertain linguistic environment," International Journal of Uncertainty, Fuzziness and Knowledge-Based Systems, vol. 17, no. 2, pp. 251-267, 2009.

[22] J. H. Park, M. G. Gwak, and Y. C. Kwun, "Uncertain linguistic harmonic mean operators and their applications to multiple attribute group decision making," Computing, vol. 93, no. 1, pp. 47-64, 2011.

[23] P. Liu and Y. Su, "Multiple attribute decision making method based on the trapezoid fuzzy linguistic hybrid harmonic averaging operator," Informatica, vol. 36, no. 1, pp. 83-90, 2012.

[24] Z. S. Xu, "Intuitionistic fuzzy aggregation operators," IEEE Transactions on Fuzzy Systems, vol. 15, no. 6, pp. 1179-1187, 2007.

[25] J. C. Harsanyi, "Cardinal welfare, individualistic ethics and interpersonal comparisons of utility," Journal of Political Economy, vol. 63, pp. 309-321, 1955.

[26] R. R. Yager and D. P. Filev, "Induced Ordered Weighted Averaging operators," IEEE Transactions on Systems, Man, and Cybernetics B, vol. 29, no. 2, pp. 141-150, 1999.

[27] Z. S. Xu, "Induced uncertain linguistic OWA operators applied to group decision making," Information Fusion, vol. 7, no. 2, pp. 231-238, 2006.

[28] Z.S. Xu, "On generalized induced linguistic aggregation operators," International Journal of General Systems, vol. 35, no. 1, pp. 17-28, 2006.

[29] G. Wei, X. Zhao, R. Lin, and H. Wang, "Uncertain linguistic Bonferroni mean operators and their application to multiple attribute decision making," Applied Mathematical Modelling, vol. 37, no. 7, pp. 5277-5285, 2013.

[30] C. Bonferroni, "Sulle medie multiple di potenze," Bolletino Matematica Italiana, vol. 5, pp. 267-270, 1950.

[31] G. Beliakov, A. Pradera, and T. Calvo, Aggregation Functions: A Guide For Practitioners, Springer, Berlin, Germany, 2007.

[32] D. J. Yu and Y. Y. Wu, "Interval-valued intuitionistic fuzzy Heronian mean operators and their application in multi-criteria decision making," African Journal of Business Management, vol. 6, no. 11, pp. 4158-4168, 2012.

[33] D. J. Yu, "Intuitionistic fuzzy geometric Heronian mean aggregation operators," Applied Soft Computing, vol. 13, no. 2, pp. 1235-1246, 2013.
[34] R. M. Rodriguez, L. Martinez, and F. Herrera, "Hesitant fuzzy linguistic term sets for decision making," IEEE Transactions on Fuzzy Systems, vol. 20, no. 1, pp. 109-119, 2012.

[35] M. M. Xia, Z. S. Xu, and B. Zhu, "Geometric bonferroni means with their application in multi-criteria decision making," Knowledge-Based Systems, vol. 40, pp. 88-100, 2013. 


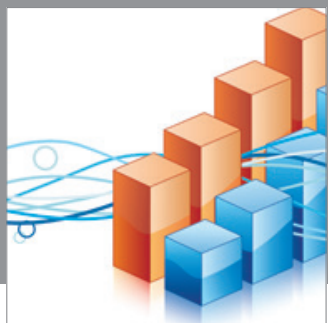

Advances in

Operations Research

mansans

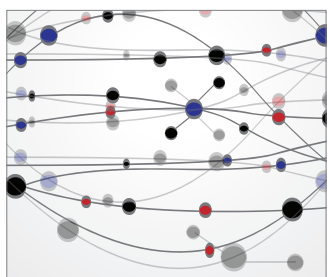

The Scientific World Journal
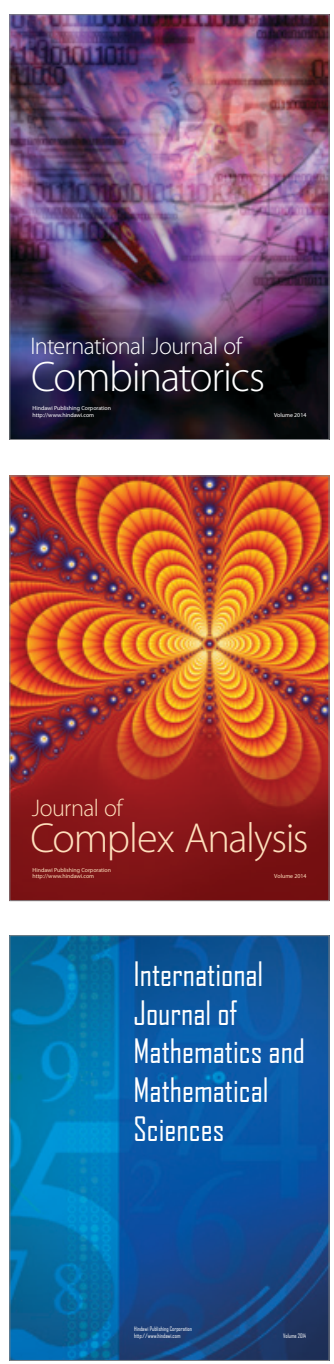
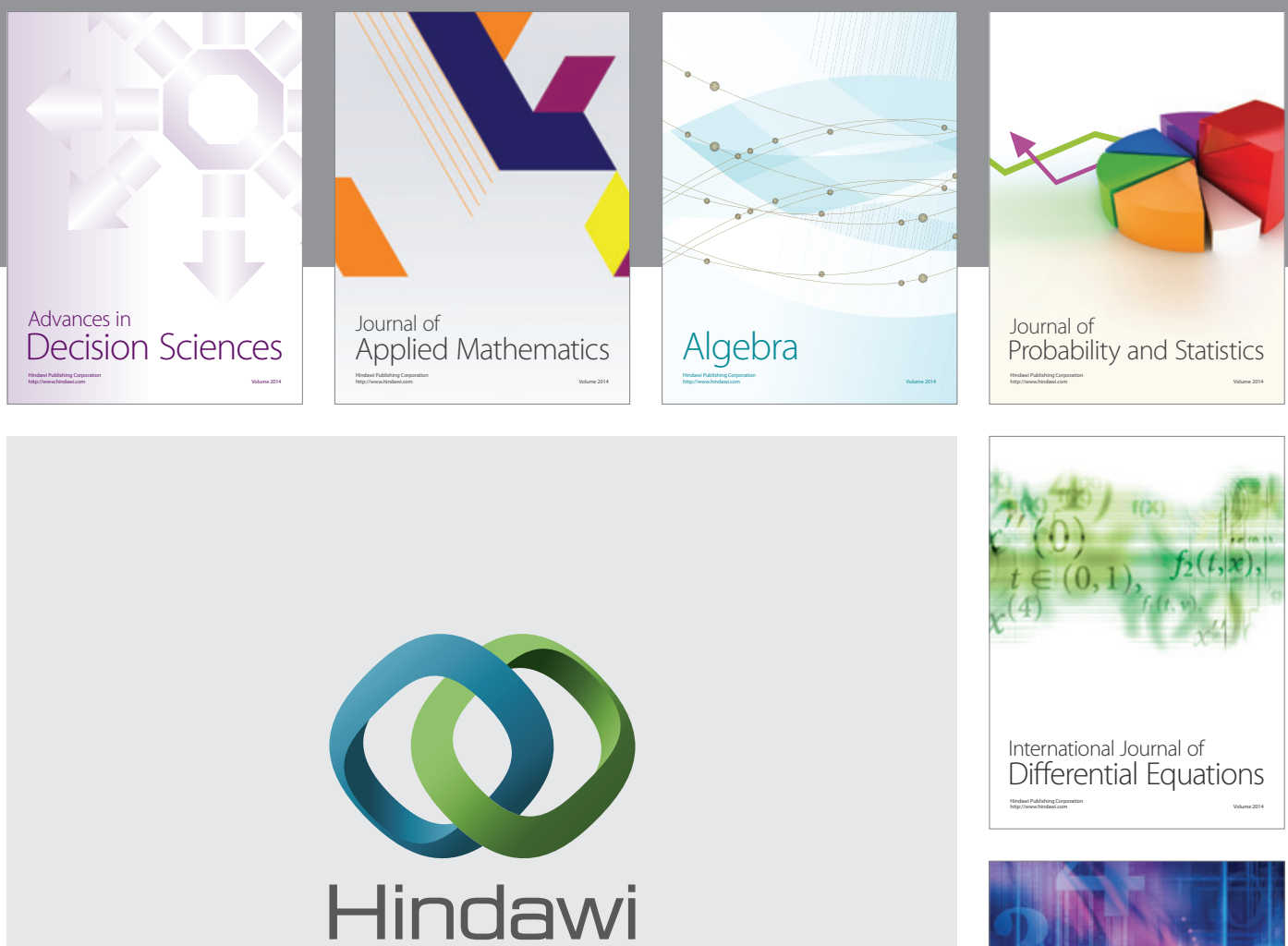

Submit your manuscripts at http://www.hindawi.com
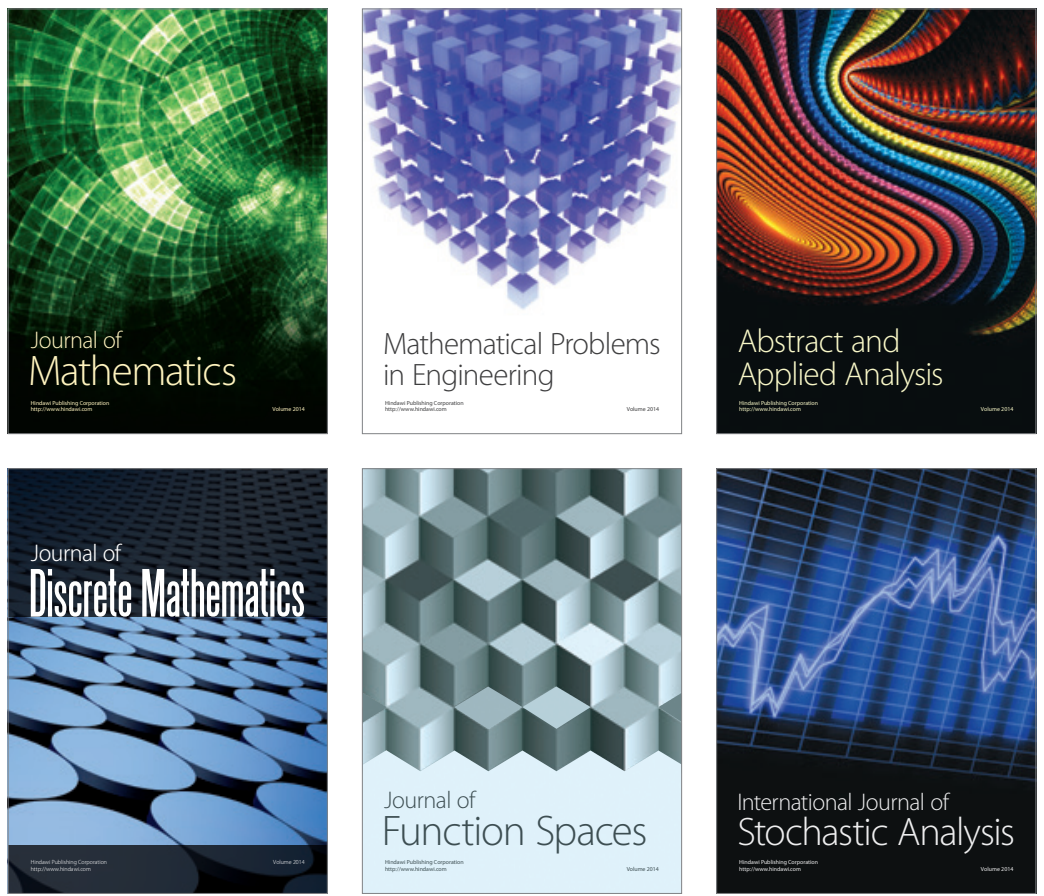

Journal of

Function Spaces

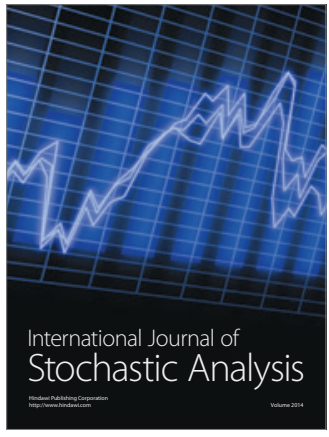

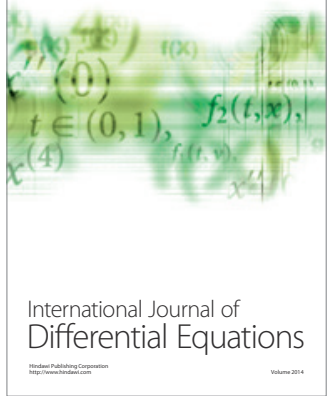
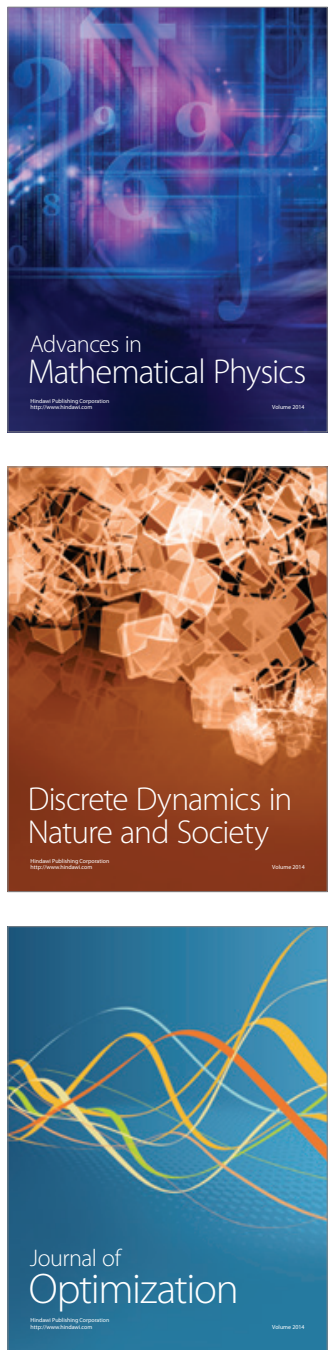